Keywords: EORTC-PAMM; CRUK; in vitro screen; pharmacological approach; anticancer drug discovery and development; PDX; European $\mathrm{NCl}$ compounds; academic platform

\title{
Pharmacologically directed strategies in academic anticancer drug discovery based on the European $\mathrm{NCl}$ compounds initiative
}

\author{
Hans R Hendriks ${ }^{*}, 1$, Anne-Sophie Govaerts ${ }^{2}$, Iduna Fichtner ${ }^{3}$, Sally Burtles ${ }^{4}$, Andrew D Westwell ${ }^{5}$ and \\ Godefridus J Peters ${ }^{\star}, 6$ on behalf of the EORTC-PAMM Group \\ ${ }^{1}$ Hendriks Pharmaceutical Consulting, J. Wagenaarstraat 67, Purmerend $1443 L R$, The Netherlands; ${ }^{2} E O R T C$, Avenue E. Mounier \\ 83/11, Brussels 1200, Belgium; ${ }^{3}$ Max-Delbrück Center for Molecular Medicine, Robert-Rössle-Str. 10, Berlin-Buch 13125, Germany; \\ ${ }^{4}$ Queen Mary, University of London, Mile End Road, London E1 4NS, UK; ${ }^{5}$ School of Pharmacy and Pharmaceutical Sciences, \\ College of Biomedical and Life Sciences, Cardiff University, Redwood Building, King Edward VII Avenue, Cardiff, CF10 3NB, \\ Wales, UK and ' ${ }^{6}$ Secretary EORTC-PAMM group, Laboratory Medical Oncology, VU University Medical Centre, Cancer Center \\ Amsterdam, De Boelelaan 1117, Amsterdam 1081 HV, The Netherlands
}

Background: The European NCl compounds programme, a joint initiative of the EORTC Research Branch, Cancer Research Campaign and the US National Cancer Institute, was initiated in 1993. The objective was to help the $\mathrm{NCl}$ in reducing the backlog of in vivo testing of potential anticancer compounds, synthesised in Europe that emerged from the $\mathrm{NCl}$ in vitro 60-cell screen.

Methods: Over a period of more than twenty years the EORTC—Cancer Research Campaign panel reviewed 2000 compounds of which 95 were selected for further evaluation. Selected compounds were stepwise developed with clear go/no go decision points using a pharmacologically directed programme.

Results: This approach eliminated quickly compounds with unsuitable pharmacological properties. A few compounds went into Phase I clinical evaluation. The lessons learned and many of the principles outlined in the paper can easily be applied to current and future drug discovery and development programmes.

Conclusions: Changes in the review panel, restrictions regarding numbers and types of compounds tested in the $\mathrm{NCl}$ in vitro screen and the appearance of targeted agents led to the discontinuation of the European $\mathrm{NCl}$ programme in 2017 and its transformation into an academic platform of excellence for anticancer drug discovery and development within the EORTC-PAMM group. This group remains open for advice and collaboration with interested parties in the field of cancer pharmacology.

\section{INTRODUCTION}

Here we describe the evolution towards a pan-European multidisciplinary academic platform for anticancer drug development from an international academic collaboration between both sides of the Atlantic. It started in 1993 as a joint initiative

of the EORTC Research Branch, Cancer Research Campaign (CRC, now CRUK) and the US National Cancer Institute (NCI). The aim was to help the NCI in reducing the backlog of potential anticancer compounds, which emerged from the NCI in vitro 60-cell line screen, awaiting in vivo evaluation. The collaboration focused on compounds synthesised in Europe

*Correspondence: Professor GJ Peters; E-mail: gj.peters@vumc.nl or Dr HR Hendriks; E-mail: hans@pharmaceuticalconsulting.nl Dedicated to the memory of Dr Angelika M Burger, Karmanos Cancer Institute, Wayne State University, Detroit, MI, USA.

Received 6 February 2017; revised 20 April 2017; accepted 12 May 2017; published online 13 June 2017

(C) 2017 Cancer Research UK. All rights reserved 0007-0920/17 
showing interesting data in the NCI screen, hence the name: the European NCI compounds programme. The purpose of this paper is to critically appraise the performance of the drug discovery programme of the EORTC/CRUK in the context of using the experience gained over 20 years in novel drug development.

The NCI provided support for screening of novel chemicals as potential anticancer compounds to researchers worldwide since 1955. Until 1985, the NCI screen relied primarily on the survival of mice bearing the murine P388 and L1210 leukaemia models, other transplantable mouse solid tumour models and human tumour xenografts (HTXs) (Plowman et al, 1997). This approach delivered only a few clinically useful drugs; mainly for leukaemia. To discover novel agents and to generate new leads active against common solid tumours, the NCI completely altered its screening programme. An in vitro panel of 60 human tumour cell lines comprising a variety of tumour types was established to test the cytotoxic activity of agents, followed by secondary testing in HTXs for selected compounds. This screen became fully operational in 1990 with a capacity of 10000 compounds per year (Boyd, 1992). The compounds tested were selected from more than 20000 investigational compounds submitted to the screen annually, based on structural diversity and novelty, and were acquired from a variety of sources worldwide. Europe contributed 20\% of these compounds via the NCI Liaison Office in Brussels, which was established in 1972, to expedite the acquisition of compounds and to act as the liaison between the EORTC and the NCI (Wittes and Yoder, 1998).

In spite of the expected few agents, the new screen was not sufficiently discriminatory. Up to 1,000 compounds per annum were selected by the NCI Biological Evaluation Committee (BEC) for in vivo testing in subcutaneous HTXs instead of relatively few agents. This posed a sizable logistical and budgetary problem for the NCI; a potentially useful new chemical entity could be in the queue for more than a year. To cope with this, the NCI therefore developed a fast and low-cost hollow fibre assay (HFA) as a preliminary in vivo screen (Hollingshead et al, 1995; Hollingshead et al, 1999). The assay does not have the drawbacks of in vitro screens: the poor predictive capacity for in vivo activity and lack of information on potential pharmacological properties that are essential to deliver sufficient drug to the target (Phillips et al, 2008). The HFA was fully implemented in 1999 and has been shown to predict for HTX activity (Johnson et al, 2001; Bijnsdorp and Peters, 2008).

In the intervening years, 1990-1999, there was an urgent need to develop new anticancer agents. Concerns on the backlog of compounds for in vivo HTX testing at the NCI in the early $90 \mathrm{~s}$ led the EORTC Screening and Pharmacology Group (SPG) to propose to take on some secondary HTX testing for the NCI. This resulted in 1993 in the initiative of the European NCI compounds programme and the development of a pharmacological approach by EORTC and CRC to select potential anticancer agents (Double, 1999). Since that time all compounds originating from Europe and referred to the BEC were candidates for studies in the programme. It should be noted that already at that time many of the HTXs available in the major testing laboratories of the SPG were in fact patient-derived xenografts (PDXs) and not HTXs derived from human tumour cell lines (Boven et al, 1992; Langdon et al, 1994). The number of available PDXs in those laboratories has considerably increased over time.

The goal was to develop the selected new chemical entities in a stepwise manner using a pharmacologically guided strategy, starting with pre-formulation, assay development, and stability studies; followed by preliminary in vivo toxicology and pharmacokinetic studies as well as mechanism of action and additional in vitro and in vivo studies.

\section{MATERIALS AND METHODS}

The CRC and EORTC Research Branch set up a Review Committee of 9 and later 14 members, all with considerable experience in experimental therapeutics and anticancer drug development. They represented EORTC committees and Research Branch groups, the CRC/EORTC/NCI Joint Working Formulation Party, selected experts and the drug development offices of EORTC (NDDO, Amsterdam) and CRC (London) as well as the NCI Liaison Office (Table 1). In addition, the Review Committee identified and approached expert EORTC and CRC investigators that could be interested in working on compounds selected by the

Table 1. Members of the CRC/EORTC Review Committee on the European $\mathrm{NCl}$ compounds programme at the start in 1993 and $1995^{\mathrm{a}}$

\begin{tabular}{|c|c|c|}
\hline Name & Period ${ }^{b}$ & Affiliation when joining the committee \\
\hline Sally Burtles & 1993-2007 & Assistant Director, CRC Drug Development Office \\
\hline Tom Connors & 1993-1996 & Chair, EORTC Research Branch \\
\hline Maurizio D'Incalci & $1993-2000$ & Chair, EORTC New Drug Development Coordinating Committee (NDDCC)/Research Division \\
\hline John Double & 1993-2003 & Chair, EORTC SPG/European NCl compounds \\
\hline Hans Hendriks & 1993-1998 & Secretary EORTC NDDCC/NDDO \\
\hline Herbie Newell & $1993-2001$ & Chair, EORTC PAMM/European NCl compounds \\
\hline Herbert Pinedo & 1993-1995 & Founder NDDO \\
\hline Susanne Radtke & 1993-2004 & $\mathrm{NCl}$ Liaison Office \\
\hline Omar Yoder & 1993-1999 & $\mathrm{NCl}$ Liaison Office \\
\hline Jos Beijnen & 1995-2004 & Member CRC/EORTC/NCI Joint Working Formulation Party \\
\hline Christian Dittrich & $1995-2000$ & Chair, EORTC Preclinical Therapeutics Models Group \\
\hline Heiner Fiebig & 1995-present & Selected expert \\
\hline Roland Henrar & $1995-1998$ & EORTC NDDCC/NDDO \\
\hline David Secher & $1995-2000$ & Director, CRC Drug Development Office \\
\hline Coen van Kalken & 1995-1998 & Director NDDO \\
\hline \multicolumn{3}{|c|}{ 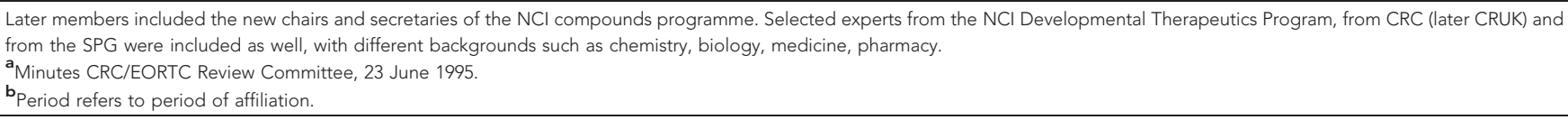 } \\
\hline
\end{tabular}


Table 2. Overview of Chairs and Secretaries CRC/EORTC Review Committee European $\mathrm{NCl}$ compounds programme

\begin{tabular}{|l|c|l|c|}
\hline Chairs & Period & \multicolumn{1}{c|}{ Secretary } & Period \\
\hline Herbie Newell & $1993-2000$ & $\begin{array}{l}\text { Hans Hendriks } \\
\text { Veronica Verdon }\end{array}$ & $\begin{array}{c}1993-1998 \\
1999\end{array}$ \\
\hline John Double & $2000-2003$ & & \\
Iduna Fichtner & $2003-2006$ & & \\
Angelika Burger & $2006-2008$ & Anne-Sophie & 2000-present \\
Frits Peters & $2008-2011$ & Govaerts & \\
Andrew Westwell & $2011-2014$ & & \\
Frits Peters (ad interim) & $2014-$ present & & \\
\hline
\end{tabular}

Review Committee. If so, they were invited to attend the Review Committee meetings. The review and discussions on the data of the compounds were strictly confidential and all involved in the evaluation and testing signed a confidentiality agreement. Table 2 shows the Chairs and Secretaries of the European NCI compounds programme over the years.

The evaluation process of the European NCI compounds started at the NCI with the collection of the available data from the BEC referrals for which permission from the suppliers was obtained to show the data to the Review Committee. The NCI Liaison Office then forwarded the data to the CRC Drug Development Office who subsequently distributed the results to the members of the Review Committee. Each member could select up to six compounds, indicate the interest in the compound on a three-point scale (borderline interesting, interesting or very interesting) and present the reason(s) for the selection. The individual evaluations were sent to the CRC Drug Development Office, which summarised the outcome and drafted the short list. At the next meeting of the Review Committee, the shortlist was discussed. The result was relayed via the NCI Liaison Office to the NCI together with a request for samples of the selected compounds. The NDDO was responsible for the distribution of the samples to EORTC and CRC investigators, and the coordination and collection of all experimental data. New experimental data were presented and discussed at the next Review Committee meeting and the decision was taken as to whether a compound qualified for further development, required further studies or was discontinued.

The data on the compounds to be judged by the Review Committee members comprised the chemical structure, the doseresponse curves over a five-log concentration range $\left(10^{-4}-10^{-8}\right.$ or $\left.10^{-5}-10^{-9} \mathrm{M}\right)$ in each of the 60 tumour cell lines after $48 \mathrm{~h}(\mathrm{~h})$ drug exposure, the mean graph, the COMPARE data and, if available, molecular target data from the NCI 60-cell line panel (Holbeck, 2004) and HFA results. Structurally related and unrelated compounds with a high degree of similarity in the COMPARE programme, the COMPARE-positive compounds (Pearson correlation coefficient $\geqslant 0.8$ ), often showed the same mechanism of action (Paull et al, 1989; Grever et al, 1992). COMPARE-negative compounds (Pearson's correlation coefficient $<0.8$ ), which did not have close matches in the COMPARE programme, were thought to be the most interesting leads by the Review Committee, as they might have a novel mechanism of action.

In summary, important selection criteria for the Review Committee were the novelty of the chemical structure, COMPARE-negativity and potency $\left(<10^{-6} \mathrm{M}\right)$ because the Review Committee was seeking compounds with a novel mechanism of action with the potential to be a clinical candidate. If enough compelling data were present analogues of existing agents and COMPARE-positive agents were occasionally considered. Differential growth inhibition and, if available, molecular target data (genes, mRNAs, proteins, and enzymes), in vivo data and the supply of material were other criteria. The NCI required for in vitro screening a minimum amount of $10-15 \mathrm{mg}$ from suppliers meaning that a very limited amount was available for the European NCI compounds programme. If the supplier was not able to provide additional material, the compound was not selected. Sometimes, for particularly interesting agents, the NCI helped by having the material manufactured by subcontractors. Figure 1 depicts the flow diagram of the selection and the development of selected European NCI compounds.

\section{RESULTS AND DISCUSSION}

The first decade (1993-2003). During the first decade of the EORTC/CRC/NCI collaboration the selected compounds were developed using Standard Operating Procedures (SOPs; Supplementary Data, SOPs A-E). The European NCI compounds programme was a voluntary system of committed investigators using their personal budgets to test the compounds. It did not receive any financial support from the EORTC, but the NCI provided limited numbers of mice for quite a number of years. The limited resources in manpower, finances, equipment and mice had to be used as efficiently as possible. Only small in vitro and in vivo studies were carried out, just sufficient to provide enough evidence to justify the next step in the preclinical development of the compound or to abandon it.

Because the solubility of many compounds is very challenging, the first stage of the pharmacological approach was pre-formulation (Table 3), followed by the development of a simple sensitive analytical assay, and stability and purity studies. The following steps aimed to determine the potential bioavailability by determination of the maximum tolerated dose (MTD, single dose (SD), and intraperitoneal (i.p.)) in mice in the best pre-formulation found, followed by plasma pharmacokinetics at various time points using the MTD. In the case where the peak plasma concentrations approached the in vitro $\mathrm{IC}_{50}$ value, the compound was further evaluated in vitro for $1 \mathrm{~h}$ and $48 \mathrm{~h}$ against the most sensitive tumour cell line in the NCI panel. The $1 \mathrm{~h}$ exposure was to mimic in vivo bolus injection and the $48 \mathrm{~h}$ to confirm the NCI data. If the compound in the $1 \mathrm{~h}$ assay was active at the concentration achieved in the pharmacokinetic study then in vivo studies were initiated against the corresponding HTX (SD, i.p.). When the compounds were inactive in the $1 \mathrm{~h}$ assay but active in the $48 \mathrm{~h}$ assay, a repeat dose schedule $(\mathrm{q} 1 \mathrm{dx} 5)$ was considered. When a compound showed interesting activity in vivo, further in vitro studies were performed to investigate whether the compound had a broader activity in other tumour cell lines/tumour types than those tested in the NCI panel and to study the haematoxicity of the compound in bone marrow using the haematopoietic stem cell assay.

The practical work of the Review Committee started in November 1993 with the review of the first set of 126 European NCI compounds. From the short-list of 31 compounds, 16 were selected. With more experience gained, the number of selected compounds of subsequent sets was reduced to 2-7 per set.

To provide an idea of the efficiency of the stepwise pharmacologically guided approach, the history of the selected compounds from the first set is given as an example. From the original 126 compounds 16 were selected, from which the development of five compounds was already abandoned after completion of the first step (pre-formulation studies) due to severe solubility or stability problems and/or impurity. Another four compounds failed to hold the threshold of the second step, the preliminary toxicology and plasma pharmacokinetic studies: one compound was still found to be unstable, one compound showed solubility and toxicity problems and the plasma level of the MTD of two compounds was below the in vitro $\mathrm{IC}_{50}$ value. In the third 


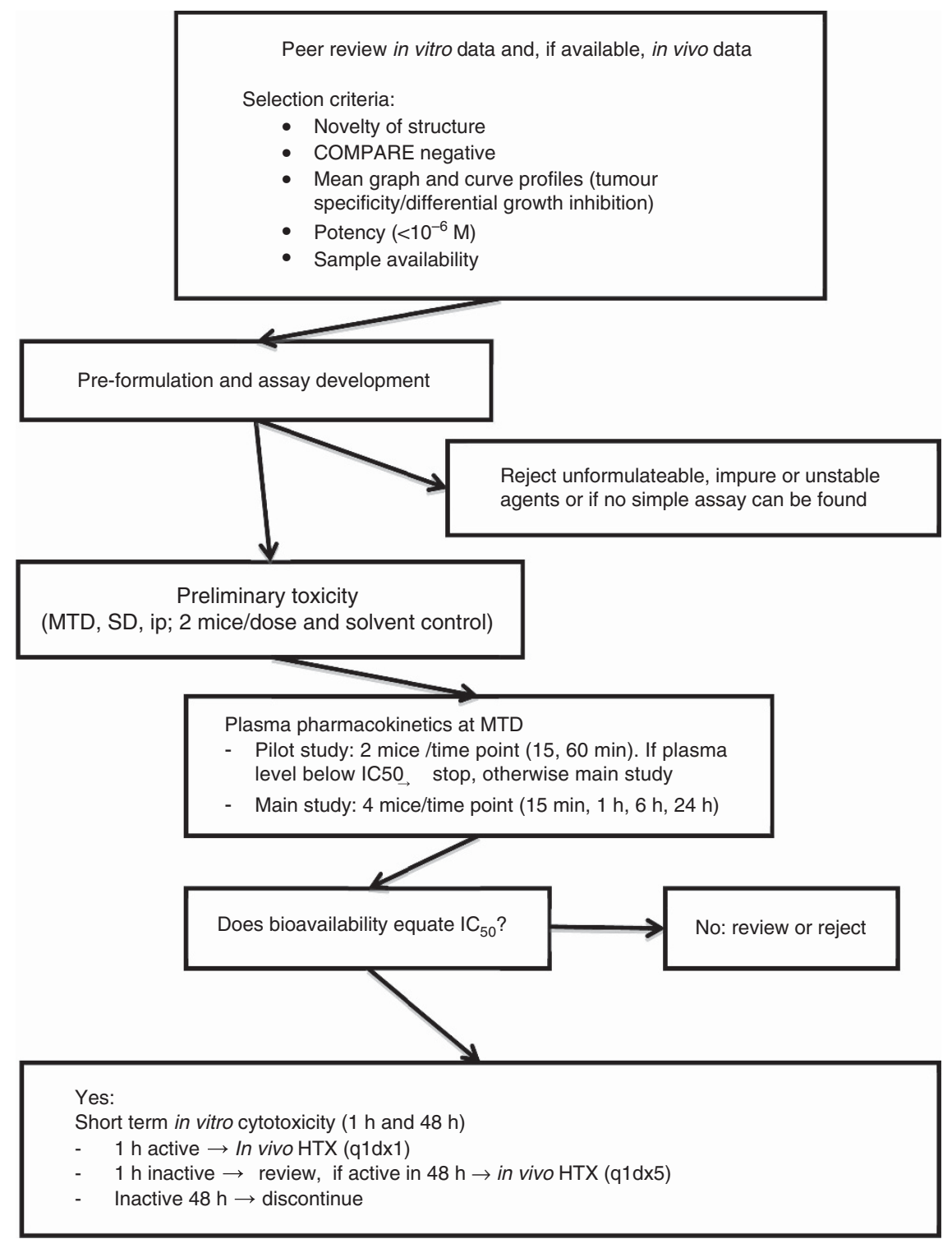

Figure 1. Flow diagram of the European $\mathrm{NCl}$ compounds programme.

Table 3. Formulations used in the European $\mathrm{NCl}$ compounds programme

\section{Formulations}

1. Water for injection

2. Absolute ethanol

3. N,N-dimethylacetamide (DMA)

4. $0.5 \%$ Tween 80 in $0.9 \% \mathrm{NaCl}(\mathrm{g} / \mathrm{v})$

5. Polyethylene glycol 400/absolute ethanol/Tween 80 (6:3:1 v/v/v)

6. DMA/Arachis oil $(1: 10, v / v)$

7. Cremophor EL/absolute ethanol $(1: 1, v / v)$

an view of bad experience with DMA as vehicle in studies in vivo, it was decided in 1995 to replace DMA by DMA/Arachis oil (1:10, v/v).

step the efficacy of the seven remaining compounds was investigated in several HTXs in vivo. Three compounds were found to be inactive in vivo, two compounds were dropped for low sensitivity of the analytical assay and a sixth compound was dropped due to lack of drug supply in combination with a low level of activity in several HTXs.

The sixteenth compound (D643314) was from the beginning considered as the most interesting one of set 1 . This COMPARE negative compound was selected for its potency $\left(\mathrm{IC}_{50}<0.5 \mu \mathrm{M}\right)$, differential activity in colon and ovarian cancer, and its chemical structure suggested to be a potential tubulin binder. It was moderately soluble in PEG 400/absolute ethanol/Tween 80 and readily soluble in $10 \%$ DMA/Arachis oil formulation. Pharmacokinetics showed a peak value at $15 \mathrm{~min} 60$ - to 90 -fold above the $\mathrm{IC}_{50}$ and at $24 \mathrm{~h}$ its plasma level was $<1 \mu \mathrm{M}$. Additional mechanistic studies (performed during in vivo evaluation) showed inhibition of tubulin assembly, substantial anti-vascular activity in vitro and activity against cisplatin-, doxorubicin- and taxolresistance human ovarian tumour cell lines in vitro. Overall, it was considered as a possible candidate for clinical phase I study. The efficacy was, however, marginally or absent in the seven HTXs tested (four ovarian, two colon, and one non-small cell lung cancer) and its was therefore concluded that were no compelling data to continue further development.

Based on the NCI selection criteria the 126 compounds of set 1 would have been tested in vivo. This overview clearly shows the strength of the pharmacologically guided approach: it rapidly removed nine out of sixteen compounds with unsuitable pharmacological characteristics. Only seven compounds went into 
in vivo efficacy studies, thereby saving animals, expenses and other resources.

Reports on the European NCI compounds programme have been presented in 1996 (Schultz et al, 1996), 1997 (Double, 1999), and 1998 (Hendriks et al, 1998). In 1998, the Review Committee had reviewed approximately 700 compounds and 40 were selected. Twenty of these compounds were dropped and the other 20 were at various stages of testing. By 2003, 1500 compounds had been reviewed and 77 selected. Most had been discontinued for reasons of solubility, instability, impurity, unavailability of material, insufficient bioavailability, lack of efficacy in HTX models in vivo, and other grounds.

Certainly, in the beginning, the European NCI compounds programme was hampered by a relatively slow transfer of information (phone, airmail, and fax) compared to today's standards, lengthy procedures in getting consent from original suppliers via NCI to share their data, difficulties in obtaining sufficient material, and resource limitations. This often resulted in a delayed start of the experiments.

Nevertheless, the merit of the European NCI compounds programme is illustrated by the development of two compounds towards clinical trials: DRH-417 and Phortress.

DRH-417 belongs to a series of pyrrolobenzodiazepine monomers, designed to fit sequence selective into the minor groove of DNA. It was very potent in the NCI 60-cell line panel (mean $\mathrm{IC}_{50} 3 \mathrm{~nm}$ ) and showed differential activity against melanoma, breast and renal cell cancer, which was confirmed by EORTC in vitro studies. DRH-417 went therefore to the next step in our pharmacological approach: investigation of its in vivo characteristics. The MTD was first established $(0.5 \mathrm{mg} / \mathrm{kg}$, SD i.p.) and plasma pharmacokinetics were determined using a novel selective HPLC method. Plasma pharmacokinetics showed concentrations far above the $\mathrm{IC}_{50}$ value. The plasma AUC was $540 \mathrm{nM} / \mathrm{h}$ and peaked at $30 \mathrm{~min}$ (171 nM), which is far above the $\mathrm{IC}_{50}$ value. Subsequently, the antitumour activity was investigated. The compounds showed marked activity in vivo in two human renal cell cancer HTXs, a breast cancer HTX and a murine syngeneic colon cancer $(P<0.01)$. Genomic profiling of in vivo sensitive HTXs demonstrated an activated insulin-like growth factor signalling pathway (Burger et al, 2007). The development of DRH-417 was however discontinued when SJG-136 (also known as SG2000) showed more promising results. SJG-136 was a novel compound in the series of pyrrolobenzodiazepine dimers, that was simultaneously developed by CRC and NCI. SJG-136 is currently in phase II clinical studies in ovarian cancer and leukaemia (Mantaj et al, 2017).

Phortress is a water-soluble benzothiazole prodrug whose active moiety (5F 203) is liberated in plasma and tumour cells. 5F 203 possessed highly potent activity (low nanomolar $\mathrm{GI}_{50}$ ) and selectivity against breast and ovarian cancer sub-panels of the NCI 60-cell line screen. 5F 203 was converted to a water soluble lysylamide prodrug to allow parenteral administration and avoidance of first pass metabolism in the liver, an abundant site of the drug activating metabolic enzyme CYP1A1. Within tumour cells Phortress is hydrolysed to 5F 203 that binds to cytoplasmic aryl hydrocarbon receptors $(\mathrm{ArH})$. The complex is translocated into the cellular nucleus, where it binds to the cyplal promoter, leading to an induction of the expression of cytochrome P450 1A1 (Figure 2). The resulting highly reactive intermediate species forms DNA adducts resulting in cell death. Based on its selective antitumour profile (breast, ovarian and renal cancers), unique mechanism of action and good pharmacokinetic characteristics, the safety and toxicity of Phortress was investigated in a phase I clinical trial in advanced cancer between 2004 and 2012 (Leong et al, 2004). Preliminary data showed that the compound was well tolerated and the MTD was not reached (Seckl et al, 2012). Thirtythree per cent of the patients (14/42) were classified as having stable disease and several cases of prolonged disease stabilisation were recorded including patients with renal cancer and mesothelioma. Despite its selective preclinical activity in breast and ovarian cancers, patients with these tumour types were not included in the study.

The development of Phortress required investigation of several successive generations of benzothiazoles (Bradshaw and Westwell, 2004). The European NCI programme contributed by studying a

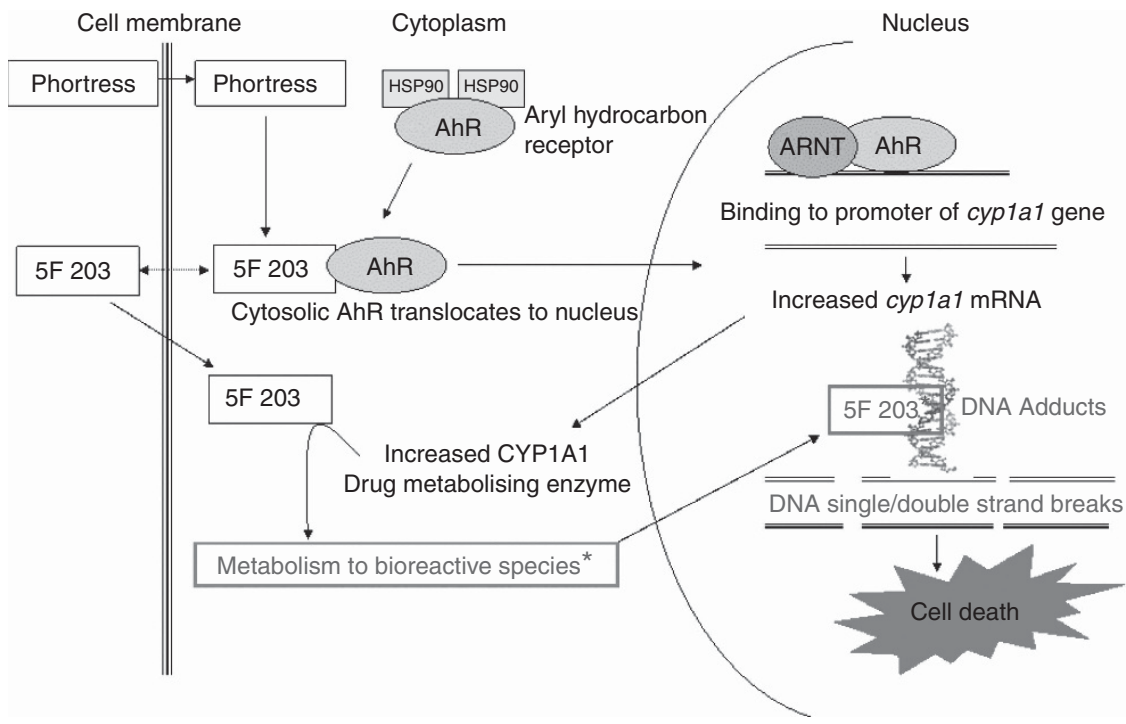

Figure 2. Self-potentiation of Phortress. After being taken up in the cell Phortress is converted in the cytoplasm to 5F203 that binds to the aryl hydrocarbon receptor. This complex is translocated to the nucleus, where it acts as a transcription factor by binding to the promotor of the cyp1a1 gene, leading to an increased cyp1a1 mRNA expression, resulting in an increased activity of the CYP1A1 enzyme that converts 5F203 into a bioreactive species that forms DNA adducts leading to single and double DNA strand break, resulting in cell death. Reproduced by kind permission of the author and Pharmaceutical-journal.com (http://www.pharmaceutical-journal.com) from Bradshaw, 2010. 
number of compounds from this programme, which increased insight into their efficacy and mechanism of action. EORTC investigators also participated in the in vivo antitumour efficacy studies of Phortress (Fichtner et al, 2004).

The second decade (2003-2013) and further. Major changes occurred in the composition and support of the work of the Review Committee in the second decade. The NDDO discontinued its support to the Review Committee in 1998 as a consequence of a difference in approach in drug development between EORTC and the NDDO (Hanauske, 1998) while in 2007 the Drug Development Office of CRUK (formed by fusion of CRC and Imperial Cancer Research Fund in 2002) withdrew from the review process due to resource limitations and the absence of clinical candidates emerging from the programme. Structural changes occurred also within the EORTC Research Division (now Translational Research Division): the PAMM group merged with the Preclinical Therapeutic Models Group (2000) and the SPG (2003). The latter operates within the PAMM as the Drug Discovery Committee (DDC) (Peters et al, 2012).

From its establishment in 1972-2001 the NCI Liaison Office in Brussels was the primary contact for the acquisition of compounds from Europe for screening by the NCI. Submission of novel agents to the NCI screen only became possible from 2001 through the NCI website of the Developmental Therapeutics programme. At the same time the NCI restricted the numbers of compounds to be tested annually from 10000 to 3000 and discontinued the screening of 'analogues related to well-studied drugs' such as anthracyclines, taxanes, camptothecins and platinum-based agents. This was changed because NCI preferred to focus on compounds with a molecular target-based rationale. In 2003 the role of the NCI Liaison Office changed to promote and facilitate the mission of the NCI Center for Global Health in the European Region and the office closed at the end of October 2015. All these changes caused a steep decline in the number of European NCI compounds referrals by the BEC to the Review Committee: up to 2004 the median number of European NCI compounds offered for review was 103 per set (range 24-230, 18 sets), but reduced to 20 per set (range 6-47, 10 sets) in the years up until 2014 with zero referrals in 2014-2016.

Economic, societal, and scientific developments in the second decade also affected the European NCI programme. Lower budgets for the laboratories of the participants and restrictions on the use of animal experiments reduced the number of experiments on compounds from the programme. This and the rising number of targeted agents necessitated a change in strategy and adaptations to the work and procedures of the European NCI compounds programme. Apart from the fact that the Review Committee was renamed the Joint EORTC Committee (JEC) on European NCI compounds, the stepwise development of compounds was abandoned in 2007 and emphasis was put on more tailored drug development plans after in-depth discussions with suppliers at the DDC meetings, which followed the JEC meetings. In addition, promising agents were further investigated in PAMM-DDC miniprojects, intended to support drug development with feeder money out of the PAMM Group income (Peters et al, 2012).

Two examples of the new approach are the developments on a series of combretastatin A-4 analogues and NSC 734237, a Paullone-like molecule.

Combretastatin A-4 is a natural product, poorly soluble, and the active metabolite of the vascular disrupting agent combretastatin A-4 phosphate. It prevents microtubule polymerisation in endothelial cells of tumour blood vessels and disrupts endothelial junctions, causing tumour blood vessel collapse and tumour necrosis (Dark et al, 1997; Tozer et al, 2002). The JEC selected a couple of COMPARE-negative compounds from a series of novel combretastatin A-4 analogues (1,5-diaryl-1H-imidazoles), based on their high potency ( $\mathrm{nM}-\mu \mathrm{M}$ range) and differential selectivity in human colon, breast and renal cancer cell lines. In the subsequent DDC meeting, the supplier presented an overview of and the background on the compounds. Subsequent studies, partly as a PAMM-DDC mini-project, focused on three compounds (NSC736359, NSC736992, NSC733436) and their water-soluble salts. The results showed that the compounds disorganised microtubules, selectively affected endothelial cell morphology and capillary formation in vitro, and caused blood vessel shutdown and tumour necrosis in vivo, thus confirming their vascular disrupting properties (Bonezzi et al, 2009). The antitumour activity (SD, i.p.) against a well-vascularised breast cancer HTX in vivo was moderate at tolerated doses but more pronounced at toxic dose levels. Additional studies with the water-soluble salt of NSC736992, the most potent one of the series, in the same HTX confirmed that its efficacy was moderate to borderline. This was also observed with other treatment schedules, while the efficacy was not increased in combination with carboplatin or taxol. In both experiments, the density (size and number) of $\mathrm{CD}_{3}{ }^{+}$blood vessels was not affected by the test compounds alone or in combination.

The Paullone-like molecule NSC 734237 is another example and part of a series of isoindoloquinoxalines with $\mathrm{IC}_{50}$ values in the low and sub $\mu \mathrm{M}$ range (Diana et al, 2008). It showed reasonable, but inconclusive, differential activity for CNS, colon, melanoma and breast cancer lines in independent assays in the NCI in vitro screen. The compound was quite insoluble (6\% active drug in a liposomal formulation). In a subsequent EORTC in vivo study it induced, however, dose-dependent antitumour activity in the doxorubicin-resistant, oestrogen receptor negative human breast cancer xenograft MT-3/ADR with significant activity at the MTD (i.p., d8-12, 15-19). Immunohistochemistry showed a dosedependent reduction in both $\mathrm{CD}_{3} 1^{+}$microvessel density and Ki-67 staining demonstrating cytotoxic activity and antivascular effects. The efficacy in the chemosensitive human breast cancer xenograft MBA-MB 453 was, however, marginal (i.p., q1dx5) or inactive (i.p., q7dx2). In the meantime results from the $\mathrm{NCI}$ Molecular Targets Laboratory showed inhibition of the topoisomerase I enzyme and inhibition of tubulin polymerisation. After consultation with the supplier, the $\mathrm{IC}_{50}$ profile of 13 other structures from the same series was investigated in a limited number of tumour cell lines in vitro. Four compounds were selected and then tested against the whole cell line panel at Oncotest in Freiburg: two had differential activity against human pancreatic cancer (1/2), non-small cell lung cancer, and melanoma tumour cell lines. Lack of material prevented plans to investigate whether the compound could overcome topoisomerase I-resistance and perhaps possess PARP inhibitory activity. In conclusion, the compound showed interesting activity and mechanisms of action, but solubility prevented further investigations. A more highly soluble analogue or a new formulation would be options to investigate.

Lessons learned and future prospects. Approximately 2000 compounds were reviewed and 95 selected in more than 20 years of the European NCI compounds programme. Several compounds moved to early stage clinical development, but no compounds progressed to late stage clinical evaluation. The pharmacologically directed approach demonstrated clearly that a few simple procedures with small numbers of mice quickly eliminated agents that were highly unlikely to have any antitumour activity, either because they were impure, unstable, unformulatable or had a low bioavailability. Existing systems would have required many more mice to produce the same result, which is not only important economically but also ethically. Double already proposed in 1999 that with adequate resourcing the process could run quite quickly and agents with unsuitable pharmacological properties could have been eliminated in less than three months. This would have represented a major overall saving in finances and animals but also 
in drug development time which would have allowed urgently needed new agents to enter clinical studies more rapidly (Hendriks et al, 1998; Double, 1999). This approach would nowadays perfectly fit within for instance the multi-tier framework proposed by the Cancer Target Discovery and Development Network, 2016 that aims to convert 'Big Data (e.g., novel sequencing platforms)' into pharmacologic targets, lead compounds, and biomarkers for rapid clinical testing.

With regard to the original goal of the initiative 'to identify new chemical entities with pharmacological properties that may predict genuine clinical potential' against solid cancers, limited success was achieved in cases such as the benzothiazole prodrug (Phortress) and the pyrrolobenzodiazepine monomer DRH-417. That is not surprising given the large attrition rate of novel anticancer agents (Kamb et al, 2007) and the number of compounds reviewed, selected and tested in the European NCI compounds programme. It is, however, important to realise as stated by JEC member Malcolm Stevens at the 2010 DDC meeting that 'a number of anticancer drugs could not have been developed without knowledge gained from work on agents that did not go into or went into clinical studies but did not make it'. The informal but confidential nature of the JEC was essential for this success as well as interactions between the various disciplines represented in the JEC. Such a multidisciplinary academic network of experts in preclinical anticancer drug development was unique in the early 1990s, even between various departments within a university, let alone between various universities in different European countries. Nowadays we can hardly imagine not collaborating in multidisciplinary teams.

In the 2nd decade of the European NCI compounds programme the pharmacologically directed approach was partially abandoned and replaced by intensive discussions with suppliers on characteristics of the compounds and the way to develop them. Unfortunately, the engagement from the suppliers was with some exceptions low. Therefore, the JEC refocused its efforts to identify and collaborate with pharmaceutical chemistry-based academic groups. These groups share a common interest in drug discovery that would benefit from interactions with JEC and the PAMM-DCC. The various panels of the early HTXs (now called PDXs) have been characterised by genomic profiling (for instance Fiebig et al, 2007, Fichtner et al, 2008). These carefully built-up portfolios of PDXs (profiled for many standard chemotherapeutic, targeted agents and investigational agents) will further contribute to the development of compounds against novel molecular targets. Development of PDXs from ethnic subtypes (e.g., China, Japan, India) could refine the test system. In addition, interactions with statisticians may contribute to coping with analysis of big data in oncology.

To summarise, the European NCI compounds programme and JEC was successful in its initial aims. The programme has now been superseded by other drug discovery approaches and therefore discontinued/terminated. However, it is important that the lessons learned from over 20 years of multinational and multidisciplinary collaborations are not lost and are applied to future drug discovery programmes. The EORTC-PAMM group (and the associated Drug Development Committee) nevertheless remains active and will provide a platform for anticancer drug discovery and development, open for advice and collaboration to academia, biotechnology and pharmaceutical industry partners.

\section{CONCLUSIONS}

In spite of the collaborative effort provided on a voluntary basis and very restricted budgets, the initial ambitious aim of the European NCI compounds initiative to develop compounds coming from the NCI screen toward clinical evaluation and implementation was partly achieved since several drugs went into
Phase I clinical trials. Development of compounds was hampered for various reasons such as speed of experimentation, frequency of the JEC meetings and absence of funding. Of utmost importance, the work resulted in fruitful multidisciplinary interactions in Europe and increased the understanding of knowledge of structures and mechanism of actions of drugs that made it to the clinic. The programme transformed from pan-European multidisciplinary collaborations into an academic platform of excellence for anticancer drug discovery and development. It fosters the collaborations with the NCI and is open for any initiative in the field of cancer pharmacology.

\section{ACKNOWLEDGEMENTS}

We acknowledge the hard work and input of all persons from EORTC, CRUK, Susanne Radtke at NCI Liaison Office and Richard Camalier and others at NCI who actively participated in this initiative since 1993.

\section{CONFLICT OF INTEREST}

The authors declare no conflict of interest.

\section{REFERENCES}

Bijnsdorp IV, Peters GJ (2008) Hollow fiber assay. In: Schwab M (ed). Encyclopedia of Cancer. Springer Verlag: Berlin, Germany, pp 1404-1406.

Bonezzi K, Taraboletti G, Borsotti P, Bellina F, Rossi R, Giavazzi R (2009) Vascular disrupting activity of tubulin-binding 1,5-diaryl-1H-imidazoles. J Med Chem 52: 7906-7910.

Boven E, Winograd B, Berger DP, Dumont MP, Braakhuis BJ, Fodstad O, Langdon S, Fiebig HH (1992) Phase II preclinical drug screening in human tumor xenografts: a first European multicenter collaborative study. Cancer Res 52: 5940-5947.

Boyd MR (1992) The future of new drug development. In: Niederhuber JE (ed). Current Therapy in Oncology. B.C. Decker Inc: Philadelphia, PA, USA, pp 11-22.

Bradshaw TD (2010) Phortress: the smart antitumour agent which induces its own metabolism. Pharm J 284: 23-24.

Bradshaw TD, Westwell AD (2004) The development of the antitumour benzothiazole prodrug, Phortress, as a clinical candidate. Curr Med Chem 11: $1009-1021$.

Burger AM, Loadman PM, Thurston DE, Schultz R, Fiebig HH, Bibby MC (2007) Preclinical pharmacology of the pyrrolobenzodiazepine (PBD) monomer DRH-417 (NSC 709119). J Chemother 19: 66-78.

Cancer Target Discovery and Development Network (2016) Transforming big data into cancer-relevant insight: An initial, multi-tier approach to assess reproducibility and relevance. Mol Cancer Res 14: 675-682.

Dark GG, Hill SA, Prise VE, Tozer GM, Pettit GR, Chaplin DJ (1997) Combretastatin A-4, an agent that displays potent and selective toxicity toward tumor vasculature. Cancer Res 57: 1829-1834.

Diana P, Martorana A, Barraja P, Montalbano A, Dattolo G, Cirrincione G, Dall'acqua F, Salvador A, Vedaldi D, Basso G, Viola G (2008) Isoindolo[2,1-a]quinoxaline derivatives, novel potent antitumor agents with dual inhibition of tubulin polymerization and topoisomerase I. J Med Chem 51: 2387-2399.

Double JA (1999) A pharmacological approach for the selection of potential anticancer agents. In Contributions to Oncology, Vol 54. In: Fiebig HH, Burger AM (eds). Relevance of Tumor Models for Anticancer Drug Development. Karger: Basel, Switzerland, pp 137-143.

Fichtner I, Monks A, Hose C, Stevens MF, Bradshaw TD (2004) The experimental antitumor agents Phortress and Doxorubicin are equiactive against human-derived breast carcinoma xenograft models. Breast Cancer Res Treat 87: 97-107.

Fichtner I, Rolff J, Soong R, Hoffmann J, Hammer S, Sommer A, Becker M, Merk J (2008) Establishment of patient-derived non-small cell lung cancer 
xenografts as models for the identification of predictive biomarkers. Clin Cancer Res 14: 6456-6468.

Fiebig HH, Schüler J, Bausch N, Hofmann M, Metz T, Korrat A (2007) Gene signatures developed from patient tumor explants grown in nude mice to predict tumor response to 11 cytotoxic drugs. Cancer Genomics Proteomics 4: 197-209.

Grever MR, Schepartz SA, Chabner BA (1992) The National Cancer Institute: Cancer drug discovery and development program. Semin Oncol 19: 622-638.

Hanauske AR (1998) NDDO splits from EORTC. Ann Oncol 9: 1145-1146.

Hendriks HR, Burtles S, Radtke S, Schultz RJ, Double JA, Sausville EA, Newell DR (1998) Cell-based screening and pharmacological strategies for the selection of new anticancer agents: A collaboration between the National Cancer Institute (NCI), Cancer Research Campaign (CRC) and EORTC. Ann Oncol 9(Suppl 2): 40, Abstract 155.

Holbeck SL (2004) Update on NCI in vitro drug screen utilities. Eur J Cancer 40: 785-793.

Hollingshead M, Plowman J, Alley M, Mayo J, Sausville E (1999) The hollow fiber assay. Contributions to Oncology. In: Fiebig HH, Burger AM (eds). Relevance of Tumor Models for Anticancer Drug Development, Vol 54. Karger: Basel Switzerland, pp 109-120.

Hollingshead MG, Alley MC, Camalier RF, Abbott BJ, Mayo JG, Malspeis L, Grever MR (1995) In vivo cultivation of tumor cells in hollow fibers. Life Sci 57: 131-141.

Johnson JI, Decker S, Zaharevitz D, Rubinstein LV, Venditti JM, Schepartz S, Kalyandrug S, Christian M, Arbuck S, Hollingshead M, Sausville EA (2001) Relationships between drug activity in NCI preclinical in vitro and in vivo models and early clinical trials. Br J Cancer 84: 1424-1431.

Kamb A, Wee S, Lengauer C (2007) Opinion-why is cancer drug discovery so difficult? Nat Rev Drug Discov 6: 115-120.

Langdon SP, Hendriks HR, Braakhuis BJ, Pratesi G, Berger DP, Fodstad O, Fiebig HH, Boven E (1994) Preclinical phase II studies in human tumor xenografts: a European multicenter follow-up study. Ann Oncol 5: $415-422$.

Leong CO, Suggitt M, Swaine DJ, Bibby MC, Stevens MFG, Bradshaw TD (2004) In vitro, in vivo, and in silico analyses of the antitumor activity of 2-(4-amino-3-methylpenyl)-5-fluorobenzothiazoles. Mol Cancer Ther 3: $1565-1575$.
Mantaj J, Jackson PJM, Rahman K, Thurston DE (2017) From anthramycin to pyrrolobenzodiazepines (PDB)- containing antibody conjugates (ADCs). Angew Chem Int Ed Engl 56: 462-488.

Paull KD, Shoemaker RH, Hodes L, Monks A, Scudiero DA, Rubinstein L, Plowman J, Boyd MR (1989) Display and analysis of patterns of differential activity of drugs against human tumour cell lines: development of mean graph and COMPARE algorithm. J Natl Cancer Inst 81: 1088-1092.

Peters GJ, Chatelut E, Larsen AK, Zaffaroni N. on behalf of PAMM (2012) EORTC-related new drug discovery and development activities: role of the Pharmacology and Molecular Mechanisms Group. Eur J Cancer. Suppl 10: 128-140.

Phillips RM, Bibby MC, Double JA (2008) A critical appraisal of the predictive value of in vitro chemosensitivity testing. J Natl Cancer Inst 82: $1457-1468$.

Plowman J, Dykes DJ, Hollingshead M, Simpson-Herren L, Alley MC (1997) Human tumor xenograft models. In: Teicher B (ed). Anticancer Drug Development Guide: Preclinical Screening, Clinical Trials, and Approval. Humana Press Inc.: Totowa, NJ, USA, pp 101-125.

Schultz R, Burtles S, Hendriks HR, Yoder O, Radtke S, Narayanan V, Sausville EA (1996) Anticancer drug discovery: the National Cancer Institute's acquisition/screening programs and collaboration with the CRC/EORTC. Ann Oncol 7(Suppl 1): 105, Abstract 366.

Seckl M, Cresti N, Boddy A, Phillips R, Chapman F, Calvert H, Robson L, Plummer R (2012) A Cancer Research UK Phase I trial of Phortress (novel antitumour benzothiazole) given intravenously in consecutive 21 day cycles with treatment on Day 1 of each cycle. In: Proc 8th. NCRI Cancer Conference, 4-7 November 2012: Liverpool, UK, Abstract LB79.

Tozer GM, Kanthou C, Parkins CS, Hill SA (2002) The biology of the combretastatins as tumour vascular targeting agents. Int J Exp Pathol 83: 21-38.

Wittes RE, Yoder O (1998) One community. Ann Oncol 9: 251-254.

This work is published under the standard license to publish agreement. After 12 months the work will become freely available and the license terms will switch to a Creative Commons AttributionNonCommercial-Share Alike 4.0 Unported License.

Supplementary Information accompanies this paper on British Journal of Cancer website (http://www.nature.com/bjc) 